\title{
Review of lens-assisted beam steering methods
}

\author{
Steven J. Spector* \\ Draper, Cambridge, Massachusetts, United States
}

\begin{abstract}
Lens-assisted beam steering (LABS) has emerged as a promising solution for compact chip-based optical beam steering for light detection and ranging (LiDAR) applications. In a LABS system, light is steered within an integrated optical chip and emitted at a desired location. This emitted light is focused out into the scene with a lens, analogous to a camera operating in reverse. LABS systems offer many advantages compared to competing technologies such as solid-state reliability, simple control, compactness, and fast random access scanning. Different methods for LABS systems are described and compared. Most LABS systems demonstrated thus far have small arrays, and therefore, only offer a relatively small number of possible beam locations. It is important to understand how these systems will scale to the much larger arrays needed for a practical LiDAR system. (0) The Authors. Published by SPIE under a Creative Commons Attribution 4.0 International License. Distribution or reproduction of this work in whole or in part requires full attribution of the original publication, including its DOI. [DOI: 10.1117/1.JOM.2.1.011003]
\end{abstract}

Keywords: LiDAR; MEMS; photonics; integrated optics; silicon photonics.

Paper 21021SSV received Jul. 30, 2021; accepted for publication Oct. 26, 2021; published online Jan. 4, 2022.

\section{Introduction}

Lens-assisted beam steering (LABS), also referred to as image-based beam steering, has emerged as a promising solution for compact chip-based optical beam steering. Such systems can meet the needs for light detection and ranging (LiDAR) and free-space optical communication applications without large mechanical systems. By offering solid-state reliability, simple control, compactness, fast random access scanning, and also being scalable to large aperture size, such systems offer many advantages compared to competing technologies.

The principle behind LABS is similar to a camera operated in reverse. In a camera, a lens is used to focus light from a point out in the world onto a focal plane. The light rays entering the lens from various locations in space are focused onto corresponding locations on a detector array (or film) at the focal plane. In LABS, the detector array is swapped for an array of emission locations, and light emitted from a location in the focal plane is directed toward a spot into space. To get light to emit from a particular location in the focal plane, waveguides are used to steer the light within the plane of a photonic integrated circuit (PIC). Different methods for steering the light on a chip (and focusing) are possible, and those will be compared in this paper. The advantages of a LABS system are that they can be all solid-state [or switched with very fast microelectromechanical systems (MEMS) devices] and can be easily controlled for robust and fast random-access scanning.

\section{Comparison of Beam Steering Methods}

Table 1 lists a number of different beamsteering methods used for LiDAR applications. The first three beam steering methods (flash, macromechanical, and MEMS mirrors ${ }^{1}$ ) are in common use today, whereas the remaining three (liquid crystal metasurface-LCM, ${ }^{2}$ optical phased arrays$\mathrm{OPA},{ }^{3} \mathrm{LABS}$ ) are emerging technologies currently under development. This list is not an attempt to be complete, but it does illustrate how LABS fits into the LiDAR technology landscape. Similarly, some of the terms are non-specific (e.g., small versus large), because there is a lot of variation between different systems within a category. There terms simply reflect the generally accepted trends.

*Address all correspondence to Steven J. Spector, sspector@draper.com 
Table 1 Comparison of LiDAR scanning methods.

\begin{tabular}{|c|c|c|c|}
\hline Method & Brief description & Advantages & Disadvantages \\
\hline Flash & $\begin{array}{l}\text { Entire FOV is illuminated at one } \\
\text { time } \\
\text { Detector array for spatial } \\
\text { resolution }\end{array}$ & $\begin{array}{l}\text { - Simple (no beam } \\
\text { scanning needed) }\end{array}$ & $\begin{array}{l}\text { - Limited range (typically } \\
<50 \mathrm{~m})\end{array}$ \\
\hline $\begin{array}{l}\text { (Gross) } \\
\text { mechanical } \\
\text { scanning }\end{array}$ & Large optic or mirror is moved & $\begin{array}{l}\text { - Good range performance } \\
\text { (often }>200 \mathrm{~m} \text { ) }\end{array}$ & $\begin{array}{l}\text { Expensive; bulky; } \\
\text { significant maintenance }\end{array}$ \\
\hline $\begin{array}{l}\text { MEMS } \\
\text { mirror } \\
\text { scanning }\end{array}$ & $\begin{array}{l}\text { Light is bounced off tilting MEMS } \\
\text { mirror }\end{array}$ & $\begin{array}{l}\text { - Low cost } \\
\text { - Small size }\end{array}$ & $\begin{array}{l}\text { - Small mirror limits optical } \\
\text { performance } \\
\text { - Acoustic coupling can be } \\
\text { a concern } \\
\text { - Steering range can be } \\
\text { limited }\end{array}$ \\
\hline LCM & $\begin{array}{l}\text { Reflection grating with variable } \\
\text { pitch }\end{array}$ & $\begin{array}{l}\text { - Robust solid state } \\
\text { scanning }\end{array}$ & - 1D scanning only \\
\hline OPAs & $\begin{array}{l}\text { Optical phase between a large } \\
\text { number of emitting elements is } \\
\text { controlled to create desired beam }\end{array}$ & $\begin{array}{l}\text { - Robust solid state } \\
\text { scanning } \\
\text { - Random access } \\
\text { scanning } \\
\text { - Very small size (no need } \\
\text { for lens) } \\
\text { - Potential for low cost }\end{array}$ & $\begin{array}{l}\text { - Very large number of } \\
\text { phase elements presents } \\
\text { a challenging control } \\
\text { problem } \\
\text { - Single (spatial and } \\
\text { temporal) mode laser } \\
\text { required } \\
\text { - PIC adds optical loss } \\
\text { - Tunable laser required } \\
\text { for } 2 D \text { steering }\end{array}$ \\
\hline LABS & $\begin{array}{l}\text { Light is routed to an emitter located } \\
\text { in the focal plane of the lens }\end{array}$ & $\begin{array}{l}\text { - Robust solid state } \\
\text { scanning } \\
\text { - Random access } \\
\text { scanning } \\
\text { - Small size } \\
\text { - Potential for low cost }\end{array}$ & $\begin{array}{l}\text { - Discrete number of beam } \\
\text { positions } \\
\text { - Single (spatial) mode } \\
\text { laser required } \\
\text { - PIC adds optical loss }\end{array}$ \\
\hline
\end{tabular}

\subsection{Methods Currently in Common Use}

Flash systems are simple systems in which there is no beam steering. Instead, the entire field is illuminated at once and a detector provides both spatial resolution and timing resolution. This done in either direct time-of-flight systems (where a fast counter is used to directly measure the time-of-flight of the light ${ }^{4}$ ) or indirect time-of-flight systems (where the outgoing light and the detector efficiency are modulated, and the phase between these modulations is measured ${ }^{5}$ ). Flash systems offer low cost and small size. However, flash systems are limited in range because they require the simultaneous illumination of a large amount of spatial area, and that area increases as the range squared.

Mechanical systems offer better range by focusing a beam (which can either be a single spot, multiple spots, or sometimes a line of illumination) and then moving that beam with a mechanical motion. Most often this is done with mirrors but can also be done with prisms, or by moving the entire optical system. ${ }^{1}$ Although these systems often offer the best achievable optical performance, their complexity, size, and cost have caused the industry to look for alternatives. The leading alternative, at the moment, is to use a MEMS mirror. ${ }^{1}$ The principle behind a MEMS mirror steering is similar to the larger mechanical systems. A beam of light is bounced off a mirror (or two), and that mirror is moved to direct the beam. As is implied by the name, the difference is that a much smaller mirror, one that can be fabricated using MEMS technology, is used. (Additionally, MEMS mirror systems generally are limited to a tilting motion, while larger mechanical systems can be designed to work by either spinning or tilting.) This allows the system to be much smaller and cheaper to manufacture. However, the smaller mirror means the aperture size of the system is smaller, sacrificing optical performance, and creating a larger beam. 
MEMS mirrors can also have limited tilt range, although recently a scan angle of 180 deg was demonstrated in a one-dimensional (1D) scanning system. ${ }^{6}$ Additionally, optical amplification can be used to increase the scan range ${ }^{7}$ at the cost of reducing the effective aperture of the system.

Both MEMS and the larger mechanical systems are limited in their scan flexibility. Because these systems always involve spinning or oscillating motions, these motions cannot be set arbitrarily, and true random access scanning is not possible. Random access scanning can be valuable in a LiDAR system because it efficiently allows the system to scan objects of interest at higher rates and/or resolution than the other parts of the scene.

\subsection{Methods under Development}

\subsubsection{Liquid crystal metasurface}

The other technologies listed on Table 1 are either under development or are just now becoming commercially available on a very limited basis. LCM is a technology being pioneered by a single company, Lumotive ${ }^{2}$ and many technical details have not been made public. The technology uses liquid crystals to tune a metasurface to create a reflection grating that can be rapidly changed (in $25 \mu \mathrm{s}$ ) to change the diffraction angle. Although this technology shows promise, the limited amount of information available makes evaluation difficult. A limitation does appear to be that only $1 \mathrm{D}$ scanning is possible.

\subsubsection{Optical phased arrays}

An OPA steers light by adjusting the phase of the light such that it adds coherently in only the desired direction. The LCM could also be considered a special type of optical phased array. OPAs can be grouped into two categories: devices that modify the phase front of a plane wave or a fully integrated device. Methods for OPAs that modify a plane wave include liquid crystals in transmission, ${ }^{8}$ MEMS devices in reflection, ${ }^{9}$ and the aforementioned LCM.

In the full integrated method, a planar integrated circuit is used to direct the light from a single source to an array of emission locations, with phase shifting elements in between. This is conceptually similar to phased arrays commonly used for RADAR steering but only recently has technology advanced enough that this is feasible with the much shorter wavelength of light. OPAs are often made in a silicon photonics platform, which is a type of PIC technology based on CMOS fabrication techniques. This allows highly accurate and highly integrated components to be manufactured in large volumes. The combination of CMOS manufacturability and no moving parts has led to significant interest in OPA development. ${ }^{3}$ However, even with the advanced manufacturing being used for these devices, OPA development is challenging.

Various challenges arise in OPAs, because the phase-front needs to rapidly change. Similar to diffraction angle of a phase grating, to steer to a large-angle requires periodicity in the wavefront on the order of the wavelength. For reasonably sized apertures, thousands of phase elements are required. A recent demonstration included a planar integrated circuit with 8192 phased array elements. ${ }^{10}$ This example steered over $100 \mathrm{deg}$ in $1 \mathrm{D}$ using the phased array. This very impressive result required 8192 DAC channels (from 8 DAC chips) consuming a total power of $2.5 \mathrm{~W}$. Even with this large number of phase elements, the phase adjustments only provide steering in 1D. A fully two-dimensional (2D) phased array is not practical. To obtain 2D steering, steering in the second dimension is provided by changing the wavelength, as is done in Ref. 10 and by many other groups such as Refs. 11 and 12. Another method to obtain 2D steering is by thermally tuning the emission angle of a grating. ${ }^{13}$ Wavelength tuned steering requires a laser to be tuned over a fairly broad wavelength range. In Ref. 10, for example, to steer by $17 \mathrm{deg}$, a laser was tuned from 1515 to $1635 \mathrm{~nm}$.

\section{Lens-Assisted Beam Steering Overview}

Figure 1 shows a simple LABS system with two emission points. In a LABS system, a PIC, usually in a silicon photonics platform, directs light from an input to specific output location. 


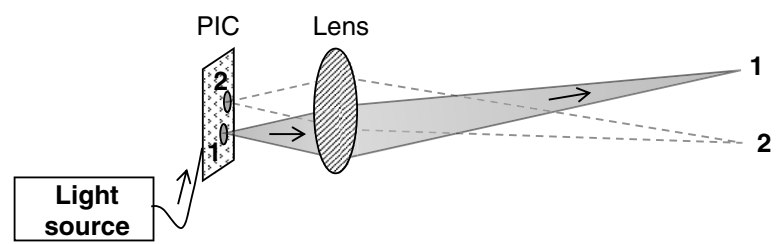

Fig. 1 A simple LABS system. The PIC directs the light to one of many possible emissions locations (here, labeled " 1 " and "2") at the focal plane of a lens.

This output location is in the focal plane of a lens. The lens creates a low-divergence beam from this smaller emission point, and the direction of the beam depends on the location of the emission.

It should be noted that there is somewhat similar beam steering technique that uses an array of vertical-cavity surface-emitting lasers (VCSELs) to emit light a selected location in a focal plane. ${ }^{14}$ For the purposes of this review paper, this is considered a separate technique and will not be covered. Generally, a limitation of the VCSEL steering method is that the brightness that can be achieved by VCSELs is insufficient for many applications.

\subsection{LABS Compared to OPA}

LABS has many similarities to an OPA, because they are both often built on a silicon photonics platform. Both technologies, therefore, share the advantages and disadvantages of this platform. As discussed, silicon photonics enables the inexpensive manufacture of extremely accurate components in high volume. In addition, these systems either use no moving parts, or, in the case of some LABS devices, MEMS structures that have extremely small excursions $(\sim 1 \mu \mathrm{m})$. This lack of a large mechanical motion makes the steering highly reliable and also makes the systems capable of rapid steering in any sequence of directions (random access). In addition to sharing the advantages, OPA and LABS technologies also share the disadvantages of the silicon photonics platform. One disadvantage is that these platforms typically only work with a single spatial mode. This limits the type of laser source that can be used. Coupling the single-mode source to the chip also poses an assembly problem, but improvements are being made. ${ }^{15}$ The other disadvantage of silicon photonics is that the components do have optical losses. Although these losses have been and will continue to be minimized with development, techniques that simply bounce light off mirrors will inherently have lower loss than a silicon photonics solution. Although additional losses are a significant disadvantage to a LABS system, a LABS system can have a much larger aperture than a MEMS mirror system, enabling more collection of light. Additionally, random access scanning in a LABS can enable more intelligent scanning of a scene, thus requiring less optical throughput.

The large advantage of the LABS approach over an OPA is that challenge of having a very large number of phase control elements, which all need to work together, is avoided. Instead of thousands of phase elements being operated at once, a much smaller number of switches can be used. Additionally, these switches can also be easier to operate, because they are binary switches, only required to operate in one of two states. Therefore, the complex analog control of an OPA can be entirely avoided. Furthermore, in the LABS approach, the emitter elements can be placed further apart. There is no requirement that all the emitter elements be placed within approximately a wavelength of each other to achieve large steering angles. This allows a much more reasonable density of elements, so fewer elements are needed, and full 2D steering is possible, with or without using wavelength tuning. It is still, however, desirable to have a relatively large number of elements, to obtain a large number of possible beam positions.

\subsection{Overview of LABS Types}

Table 2 lists and compares the different LABS systems that have been demonstrated in the literature. The different LABS systems can be distinguished by the different types of switches used, how the switches are arranged, and in one case, the use of a planar-on chip lens rather than an 
Table 2 Different published LABS systems.

\begin{tabular}{|c|c|c|c|c|c|c|}
\hline Reference & $\begin{array}{c}\text { Switch } \\
\text { arrangement }\end{array}$ & Switch type & $\begin{array}{l}\text { Wavelength } \\
\text { steering }\end{array}$ & $\begin{array}{l}\text { Waveguide } \\
\text { material }\end{array}$ & $\begin{array}{l}\text { Chip area } \\
\text { per switch } \\
\left(\mathrm{mm}^{2}\right)\end{array}$ & $\begin{array}{c}\text { Chip area } \\
\text { per beam } \\
\text { location }\left(\mathrm{mm}^{2}\right)\end{array}$ \\
\hline Li et al. ${ }^{16}$ & Matrix & $\mathrm{MZI}$ & No & Silicon nitride & 0.1 & 0.13 \\
\hline Li et al. ${ }^{17}$ & Matrix & MZI & Yes & Silicon nitride & 0.1 & 0.02 \\
\hline Ito et al. ${ }^{18}$ & Matrix & MZI & Yes & Silicon & 0.02 & 0.003 \\
\hline Lopez et al. ${ }^{19}$ & Matrix & MZI & Yes & Silicon nitride & $\begin{array}{l}\text { Component } \\
\text { sizes not } \\
\text { given }\end{array}$ & \\
\hline Inoue et al. ${ }^{20}$ & Bus & Ring resonator & No & Silicon & 0.004 & 0.0043 \\
\hline Chang et al. ${ }^{21}$ & $\begin{array}{l}\text { Matrix and } \\
\text { bus }\end{array}$ & $\begin{array}{l}\text { MZI and ring } \\
\text { resonator }\end{array}$ & No & Silicon & 0.000011 & $\begin{array}{l}\text { MZI size } \\
\text { not given }\end{array}$ \\
\hline Zhang et al. ${ }^{22}$ & Bus & $\begin{array}{l}\text { MEMS } \\
\text { waveguide }\end{array}$ & no & Silicon & 0.01 & 0.018 \\
\hline Cook et al. ${ }^{23}$ & Bus & MEMS grating & No & Silicon nitride & 0.00063 & 0.01 \\
\hline
\end{tabular}

external one. Additionally, some methods use wavelength steering for one direction and switched steering for the other direction. All the systems in the list involve an off-chip source of light that is routed to an emission location on a PIC. That PIC is placed at the focal plane of a camera lens to provide beam-steering, analogous to a camera in reverse, as described previously. In the following sections, the different LABS systems and their advantages and disadvantages will be discussed in more detail.

\section{Design Considerations}

\subsection{Chip Area Required for Each Beam Location}

An important characteristic of a LABS system is that its switched nature only enables a finite number of beam positions. Therefore, to achieve a high-resolution image, a LABS system requires either a very large number of switches, or the switched steering needs to be augmented with another steering method. Table 2 lists the size of the switches in a number of current LABS system publications. Generally, Mach-Zehnder interferometer (MZI) switches are the largest, MEMS switches are smaller, and ring-resonator switches can be the most compact. In addition to size, there are other considerations in switch choice, and further details on the different switches will be discussed in Sec. 5 .

To augment switched steering, some LABS systems use wavelength steering for 1D of steering, similar to what is done in OPAs. This allows many more beam positions, since switched steering is only necessary for one of the two directions of steering. For these types of system, it is not critical to have a very large number of switches. In Table 2, the required chip area for each possible beam position is given for the set of publications. This chip area includes the area required by the gratings and switches. Not included is the area required by waveguides used for light routing. It should be noted that all these technologies are in the early stages of development, with relatively small pixel numbers. From this table, it can be seen that addition of wavelength steering enables very good beam position density even with large switches. More detailed discussion will be provided in Sec. 5 on how each technology might scale to the much larger pixel numbers desired in a LiDAR system.

Note that to fairly compare methods that use wavelength steering with the other methods, it was assumed that wavelength tuning would create a 2D array of square pixel locations, with the size of the pixels determined by the LABS steering, and FOV equal to what was demonstrated in 
this paper. For example, in Ref. 18, the FOV was $40 \mathrm{deg} \times 4.4 \mathrm{deg}$ with 16 positions available by LABS steering. Therefore, the minimum symmetric pixel size is $0.3 \mathrm{deg} \times 0.3 \mathrm{deg}$, and for the purposes of Table 2, this device can steer to array of $134 \times 16$ symmetric positions. (It is possible to steer more finely than this in the wavelength steered direction, but generally a LiDAR system should have something close to square pixels. Therefore, square pixels were chosen to provide a reasonable comparison.)

\subsection{Source to Lens Coupling}

Another consideration for a LABS system is image formation or beam quality. As mentioned previously, a LABS system is analogous to a camera in reverse. However, the imaging properties of a LABS system are not identical to that of a typical camera. In a camera, the detector elements are generally not sensitive to the angle of the incident light rays, and any light reaching the detector element will be detected with relatively similar efficiency. Therefore, the entire aperture of the lens can be considered equally in the image formation process. In most LABS systems, a grating on the chip emits light from the plane of chip toward the lens. The effective aperture in a LABS system is not determined by solely the lens but instead by the overlap of the aperture of the lens and the illumination of the aperture by the grating.

If we consider the case of an ideal lens, an ideal design for a grating would illuminate the lens such the all the light completely fills the clear part of the aperture, and no light is outside of the clear aperture. This means that the grating mode (the near-field emission pattern) should, therefore, be an Airy disk, (the Fourier transform of the lens aperture). In practice, this is impossible with a finite grating, and so a design must find the best compromise between overfilling the aperture (and losing light) and underfilling the aperture (and creating a more divergent beam). In general, a more divergent beam is more tolerable than lost light, so it is better to underfill the lens aperture than overfill it. Additionally, different parts of the focal plane may require different illumination patterns to more ideally fill the lens aperture from different angles. Therefore, it may be advantageous to use different grating designs at different locations in the focal plane.

The grating is an important component for integrating photonics, and there is extensive literature on the optimization of gratings for different needs, such as fiber coupling, ${ }^{15}$ directly creating a focused beam, ${ }^{24}$ or emitting a beam with low divergence. ${ }^{25}$ Much of that work has been adapted for the optimization of grating for a LABS application. The pattern of the light emerging from the grating will depend on the grating design, but, in general, a smaller grating will emit a more divergent beam, fundamentally limited by the minimum divergence for a beam emerging from an aperture the size of the grating $(\delta \theta \sim \lambda / D$, where $\delta \theta$ is the angular divergence, $\lambda$ is the wavelength, and $D$ is the size of the grating). The mean emerging angle from the grating will not necessarily be oriented normal to wafer, and in fact, designing gratings that emit normal to the wafer can be a challenge. ${ }^{26}$

Not all LABS systems use the combination of a grating and a lens for beam formation. Lopez et al. ${ }^{19}$ used a planar (on-chip) lens for $1 \mathrm{D}$ steering that is in light the path prior to the grating used to emit light from the plane of the chip. Cao et al. ${ }^{27}$ used a fiber array that is edged coupled to a chip, to convert a 1D array at the edge of the chip to a 2D array of emitters. Although these methods do not use a grating, the beam formation process still involves the illumination of a lens aperture with an emission source, and many of the considerations mentioned above are the same.

\subsection{Waveguide Material}

In the LABS systems in Table 2, there are two types of waveguide materials that have been used in the LABS system, silicon, and silicon nitride, and each has its own advantages and disadvantages. The higher thermo-optic coefficient of silicon, and the conductive properties of Si, allow much more compact and efficient thermo-optic switches to be made in silicon. ${ }^{28,29}$ The higher refractive index of silicon allows more tighter bending radii, and therefore some devices, such as ring resonators, can be much smaller. Silicon nitride, on the other hand, has a broader transparency window and is transparent at shorter wavelengths, such as $905 \mathrm{~nm}$, where single-photon avalanche photodiode arrays are available. Silicon nitride is also generally not affected by two photon absorptions and can therefore handle much higher optical powers than silicon. 


\subsection{Blind Spots}

Another concern in a LABS systems can be the existence of blind spots. In a well-focused optical system with low aberrations, the beam locations created by a LABS system will not overlap. This can be understood by the fact that the lens will reproduce a highly demagnified version of the focal plane in the far-field, and the emission spots in the focal plane do not overlap. When scanning with LABS system, it is possible that small objects could be missed entirely if they are located in between the beam locations. A simple solution for this problem is to defocus the system, such that the beam locations are overlapping. ${ }^{16}$ This solution is perhaps less than ideal, because the lens of the system is required to be fairly high power (low $f / \#$ ) to capture all the light rays emitted from a divergent source, but that higher optical power results in no additional performance. A possible solution would be the addition of a lens array. This would create effective emission sources that nearly overlap and are much less divergent, enabling the use of a slower lens. Another solution to avoid blind spots is to add another steering mechanism, such as a fast steering mirror. ${ }^{30}$ The mechanical steering mechanism provides a small amount of steering to access locations in-between the nominal LABS beam steering locations. This hybrid approach of combining a mechanical steering method with the solid-state steering method does reduce the all solid-state benefit of a LABS system. However, the mechanical requirements of a system that only needs to steer over a fraction of a degree are greatly reduced compared to a system that needs to steer over a full field of view.

\subsection{LABS as a Receiver}

All of the above discussion pertains to the use of a LABS system to transmit a beam. Most of the publications, to-date, have focused on the use of LABS system as a transmitter only. However, in Ref. 27, a LABS device was used simultaneously as a transmitter and a receiver. Rather than use gratings for the outputs of LABS chip, as is typically done, in this work, the chip is coupled to a fiber array. Although it is not discussed in this paper, this choice may have been made because of the mode matching necessary in a LABS receiver.

When a LABS system is used as a receiver, the process of coupling the light into a grating from a lens is different from a typical detector, which incoherently collects all light incidents on the detector. In a LABS system, the light couples into the grating (or in the case of Ref. 27, a fiber), with an efficiency determined by the mode overlap between the focused light and the mode of the grating (or fiber). Similar to the transmit case above, an ideal grating mode for an ideal lens is an Airy disk. However, unlike the transmit case, any mode mismatch, regardless of whether it corresponds to overfilling or underfilling of the lens will result in poorer coupling and optical loss. (Note that underfilling on the transmit side will lead to larger beam, which results in lower brightness at the target.)

\section{Comparison of Different LABS Systems}

\subsection{Switched MZI Matrix}

The first type of LABS system to describe in detail is that of Li et al. ${ }^{16}$ Figure 2 gives an overview of this method. A matrix of MZI-based switches is used to guide the light to one of 16 waveguides. An MZI is a device that uses interference to control the coupling of light from one or two inputs to two outputs. An MZI can be freely adjusted to direct light to either output or split the light in any ratio. In this system, the MZI is used to put all the input light completely in one or the other output waveguides. Each waveguide at the end of the switch matrix is connected to one of the output gratings arranged in a $4 \times 4$ array, where the light will emit from the plane of photonics chip, determining the emission location. As described above, another variation of this device was demonstrated in Ref. 27, where instead of using on-chip gratings, the switch matrix was coupled to a fiber array. The waveguides are made of a silicon nitride ( $\mathrm{SiN}$ ) core surrounded by a $\mathrm{SiO}_{2}$ cladding. To control the MZI switches, heaters made of $\mathrm{Ti}$ are used to change the temperature of the waveguide. The temperature change induces a phase shift of the light by the thermo-optic effect. 


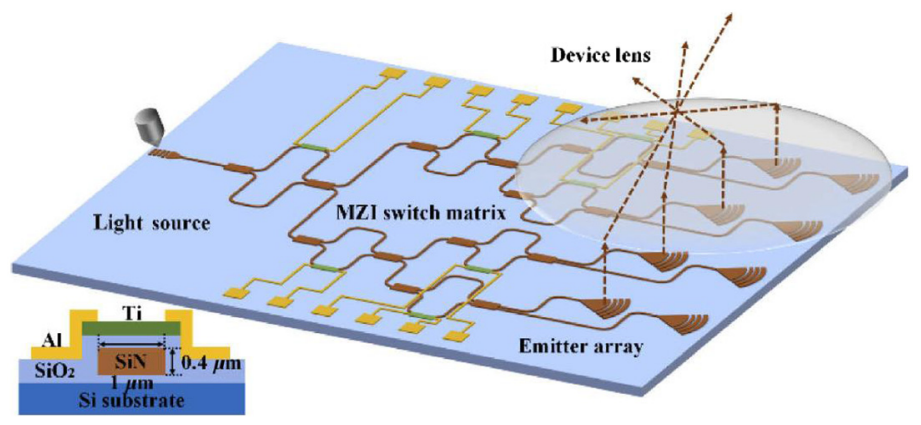

Fig. 2 Schematic of the device, reproduced with permission from Ref. 16. An MZI switch matrix is used to direct light to one several output gratings. Inset: cross section of the device with Ti heater.

One advantage of this method is that all the necessary components are readily available in a standard silicon photonics process. ${ }^{31}$ Another advantage is that only one switch at each matrix stage needs to be operated at a time, and the number of matrix stages necessary is $\log 2(N)$. Therefore, for an array with $N$ elements, only $\log 2(N)$ switches are operated at once. Similarly, the light only has to pass through $\log 2(N)$ switches, which can help minimize the optical loss.

There are several disadvantages to this method, though. The MZI switches use a considerable amount of power, requiring $88 \mu \mathrm{W}$ for the $\pi$ phase shift necessary to switch the path of the light. The losses of each MZI are significant, too with a loss of $0.5 \mathrm{~dB}$. Even with the efficient $\log 2(N)$ scaling, the amount of power consumption required and optical loss incurred become prohibitive for the very large arrays desired in a LiDAR system. Additionally, these switches are quite large (250- $\mu \mathrm{m}$ long), making such large arrays impossible in a reasonably sized chip. As discussed in Sec. 4.3, switching to a silicon waveguide platform, rather than silicon nitride, could allow the thermal switches to be more compact and efficient, but there are downsides to that approach.

\subsection{Switched Matrix with Wavelength Steering}

Another LABS method that uses a matrix of MZI switches is shown in Fig. $3 .{ }^{17}$ This work also uses $\mathrm{SiN}$ waveguides with heaters to thermo-optically switch the path of the light. Also similar to Ref. 16, this switch matrix also has 16 output ports. However, instead of small gratings that couple to a round lens, longer gratings that couple to a cylindrical lens are used. The longer grating emits a low-divergence beam that does not need further focusing from the lens. Hence, a cylindrical lens that does not focus along this direction can be used. Additionally, gratings have a wavelength-dependent emission angle, allowing steering in this direction by changing the wavelength. Thus, this method allows steering in two orthogonal directions by two mechanisms. In the $x$ direction, steering is done by directing light to a particular waveguide at the focus of the
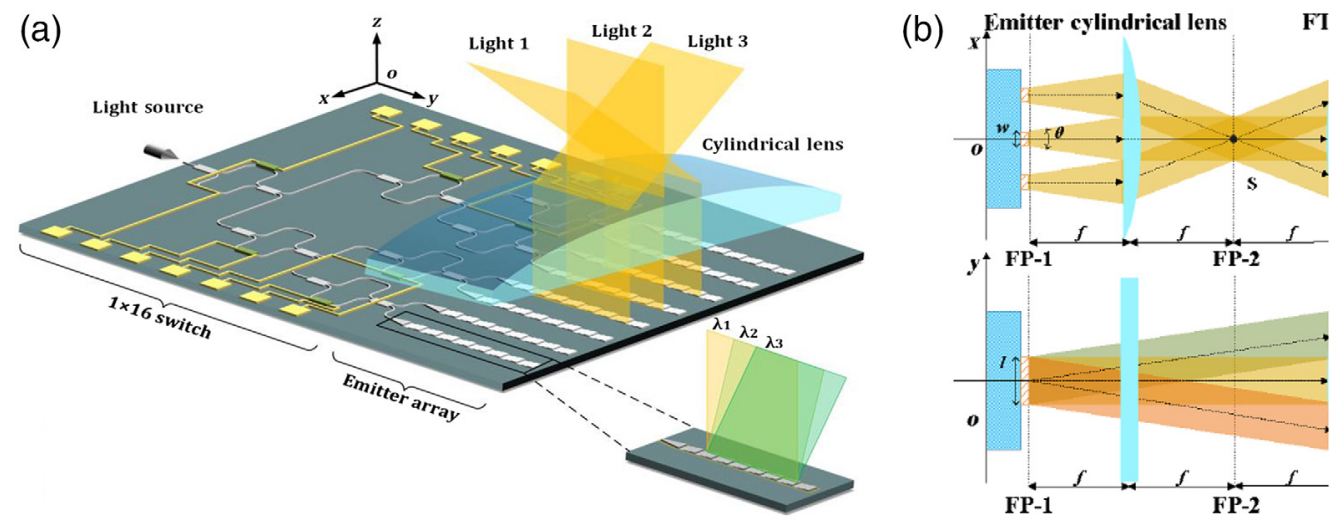

Fig. 3 Schematic of the device, reproduced with permission from Ref. 17. (a) An MZI switch matrix is used to direct light to one several long output gratings. (b) The choice of grating steers in the $x$ direction, and changing the wavelength steers in the $y$ direction. 
lens. In the $x$ direction, steering is done by altering the wavelength, which changes the emission direction of the grating. This is similar to the wavelength steering method commonly used in OPAs as described above. The work in Ref. 17 demonstrated a $16 \times 11$ spot array over a $15 \mathrm{deg} \times 12 \mathrm{deg}$ field of view. This is a fairly modest array size. However, this method has the potential to scale to a large array of beam positions, because each of the two steering methods only has to cover one axis of possible positions.

One disadvantage of this method is that only a limited steering range can be achieved with a typical grating, and a large amount of wavelength tuning is necessary. In Ref. 17, to achieve the $12 \mathrm{deg}$ of steering range, the wavelength was changed from 1456 to $1556 \mathrm{~nm}$. To improve this, the work in Ref. 18 replaces the grating with a lattice-shifted photonic crystal waveguide (LSPCW). The advantage of LSPCW structure is that there is a greater change in angle emission with changes in wavelength, enabling a larger range of steering. Also because of the symmetry of the structure, it is possible to get twice the angular coverage through switches that control the direction of light entering the LSPCW. A complication of the LSPCW is that it does not emit well at angles normal the substrate. To obtain coverage of those angles, the lens design also incorporates prisms to shift the direction of the beam toward the normal of the chip (see Fig. 4). In this work, the beam was scanned over a range of $40 \mathrm{deg}$ in the wavelength scanning direction, while

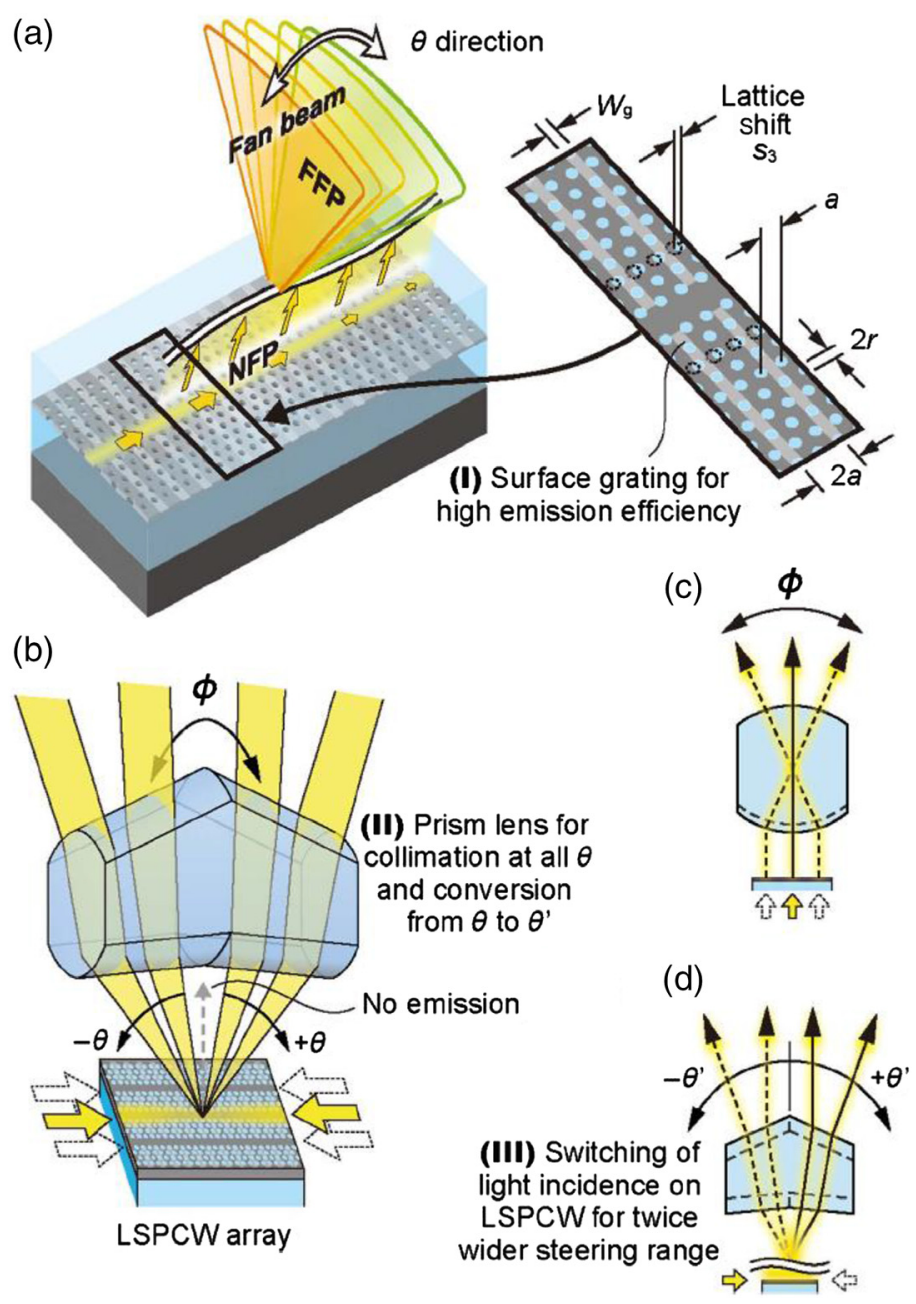

Fig. 4 Schematics of a slow-light beam steering device, reproduced with permission from Ref. 18. (a) LSPCW emits in a fan beam, with a direction $\theta$, that depends on the wavelength of light. (b) Prism lens. (c) In the $\varphi$ direction, the lens focuses the fan beam, and steering is done by selecting one LSPCW from an array. (d) In the $\theta$ direction, steering is done by changing the wavelength. Because the LSPCW cannot emit to angles near normal, a prism is used redirect the light to a more normal angle $\theta^{\prime}$. 


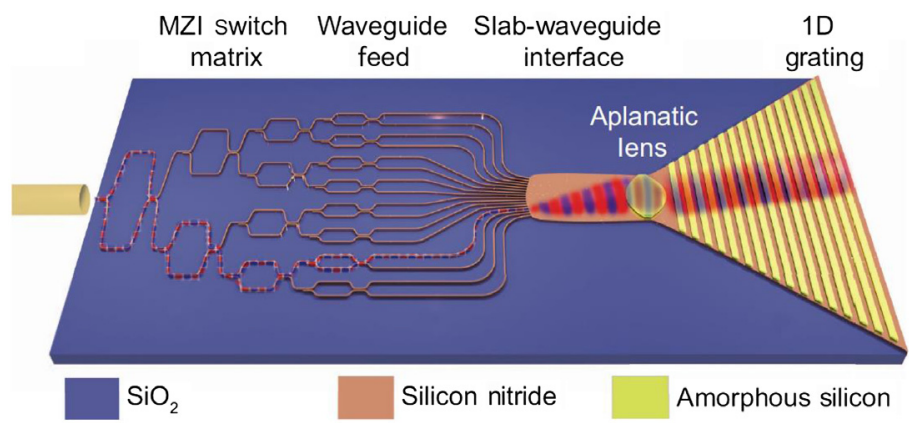

Fig. 5 Schematic of the device, reproduced with permission from Ref. 19. As in the previous method, an MZI switch matrix is used. An on chip aplanatic lens is used for focusing in one direction. The 1D grating directs light out the chip with an angle that depends on the wavelength of light.

changing the wavelength from 1525 to $1545 \mathrm{~nm}$. In the beam-switched direction, the beam was steered over 4.4 deg with 16 possible locations.

Another LABS system that uses wavelength steering is shown in Fig. $5 .{ }^{19}$ In this example, an aplanatic lens built into the chip is used to focus in $1 \mathrm{D}$. This lens comes before the grating in the path of the light. An advantage of this design is that the on-chip lens means there are fewer components in the system that need to be fabricated, assembled, and aligned. This makes this system more compact and possibly less expensive, although the on-chip lens does take up valuable space on the chip. In this work, in one direction, the beam could be steered to 16 beam positions over a 38.8-deg FOV, and in the wavelength scanning direction, the beam was scanned over a range of $12 \mathrm{deg}$, by changing the wavelength from 1500 to $1600 \mathrm{~nm}$.

A disadvantage of using wavelength-dependent steering is that it requires a tunable laser. This adds cost and complication to a LiDAR system. In Ref. 32, however, steering with an LSPCW was also demonstrated using thermo-optic tuning or electro-optic turning, rather than changing the wavelength. The amount of power required was considerable, though, with $1.3 \mathrm{~W}$ required for the electro-optic method, and $4.8 \mathrm{~W}$ required for thermo-optic tuning.

\subsection{Bus Waveguide with Switches}

Rather than a single-switch matrix, switches can also be arranged using an input waveguide bus with switches that ether direct the light off of the bus to another path (on-state) or allow the light to continue on the bus (off-state). Figure 6 shows how a series of these switches can be arranged in a $2 \mathrm{D}$ array. ${ }^{22}$ This particular work uses MEMS switches, but ring resonator switches have also been used. This is very efficient method for a compact 2D arrangement, as it minimizes the routing between the last switch and the output grating. Another characteristic of this routing method is that the light passes through only two "on" switches. If the default state of the switch

(a)

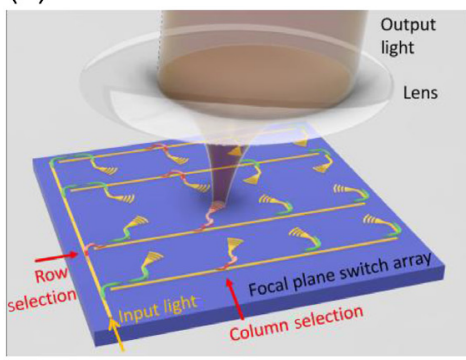

(b)

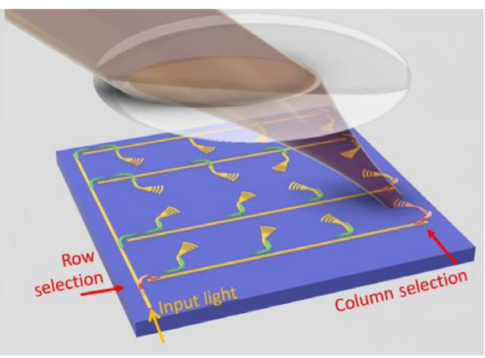

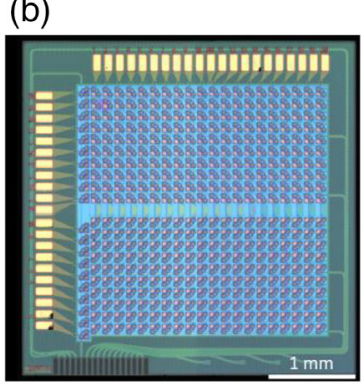

Fig. 6 Beam steering method, reproduced with permission from Ref. 22. (a) This beam steering method uses a bus waveguide with MEMS switches that can direct the light off the bus waveguide onto another waveguide. This is done using a sequence of two buses for row and column selection. (b) Microscope image of the fabricated $20 \times 20$ focal plane switch array on a $3.8 \times 3.8 \mathrm{~mm}^{2}$ chip. 
is "off," then only two switches need to be activated at a time, making control fairly simple. Additionally, the minimal number of "on" switches means a moderate amount of optical loss of an "on" switch state may be tolerable. Conversely, the light passes through a very large number of "off" switches. It is, therefore, critical that the optical loss of an "off" switch be very low.

\subsubsection{Ring resonator switches}

One example of a LABS system using ring resonator switches and a waveguide bus is shown in Fig. 7, as demonstrated in Ref. 20. Ring resonators are a wavelength sensitive device, commonly available in most silicon foundry processes. ${ }^{31}$ In a ring resonator, the light will transfer from the input bus to the output waveguide only when the light has a wavelength that is resonant with the ring. ${ }^{33}$ Off resonance, the light will stay on the bus waveguide. This allows a ring resonator to be used as a switch by tuning the ring resonator on and off resonance from the incident light. The resonant frequency can be changed with thermo-optic tuning, similar to an MZI, however, much less power is needed. Once light is switched to another waveguide, the waveguide then connects to an output grating in the usual manner. In this particular work, only a single input bus was used.

In other work (Refs. 34 and 35), a series of switches were used to enable row and column selection, similar to that shown in Fig. 6. In Ref. 34, all the switches are ring resonators, and the final ring resonator switches are coupled to gratings located directly adjacent to each ring resonator. In this work, the additional capability of directing four beams from four inputs was added. This was done using an additional layer of switches, along with the first waveguide bus being duplicated 4 times. In different works by Ref. 35, ring resonators switches were used in a 2D array, however, the output from the second switch was directed to a 1D array of gratings. This allows for high-resolution 1D scanning rather than 2D scanning.

Another method for row-column selection is shown in Fig. $8 .{ }^{21}$ In this work, the two switching geometries are combined: a switch matrix of MZIs steers light that feeds one of a number of bus waveguides with ring resonator switches. The MZI matrix steers in 1D, whereas the ring resonator switches steer in the other dimension. For a given number of outputs, the MZI matrix is much larger and requires more power. However, because the MZI only requires 1D control, significantly fewer switches of this type are needed, and the larger size is not a significant limitation.

Ring resonators can be very compact, but to make the system even more compact, in Ref. 21, angular gratings were made in the sidewalls of the rings. This causes the rings to diffract light into free space and act as emitters. Thus the functionality of the grating is added to the ring resonator. This enables a very compact area per element. The ring emitters are spaced by $7 \mu \mathrm{m} \times$ $15 \mu \mathrm{m}$ and are the most compact switch and emitter used in a LABS system. (Note that the area calculation in Table 2 does not include the MZI area, since this was not given in this paper.)

(a)

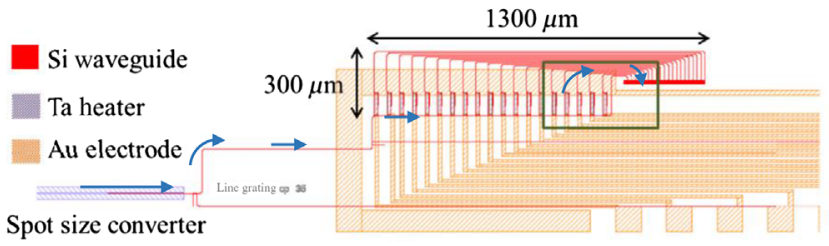

(b)

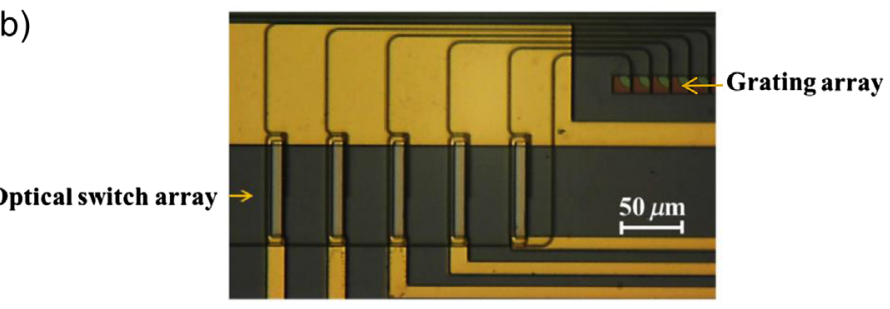

Fig. 7 Ring resonator switches, reproduced with permission from Ref. 20. (a) CAD drawing showing the chip layout. Blue arrows highly the path of the light. A bus waveguide is coupled to a linear array of ring resonator switches. (b) Inset shows an optical image of the highlighted area. 


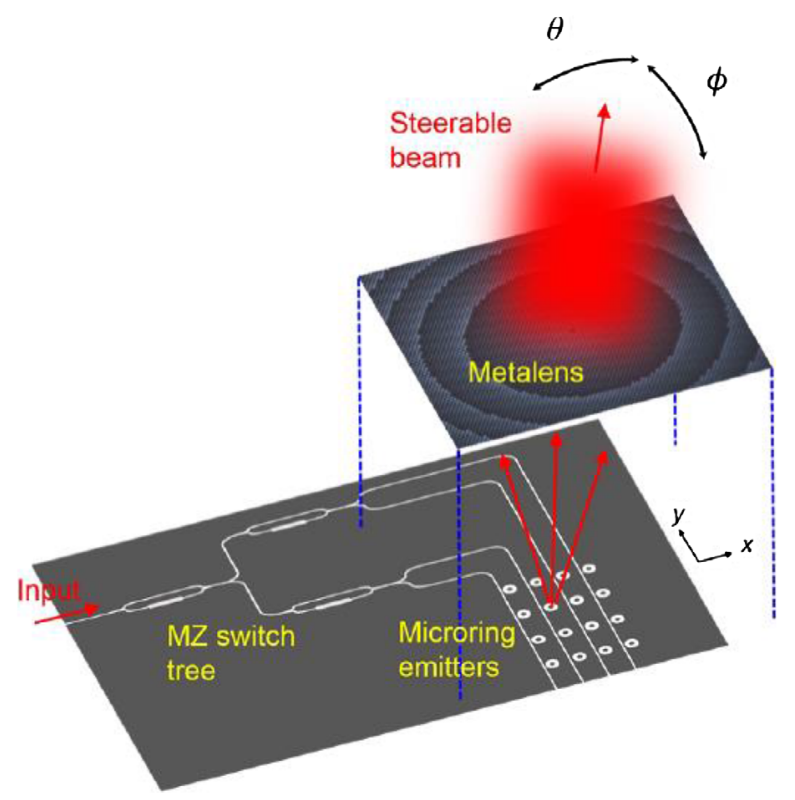

Fig. 8 Combined MZI switch matrix with ring resonator switches, reproduced with permission from Ref. 21. This work also used a metalens, a type of flat lens, instead of a more typical glass lens.

Another element of the work in Ref. 21 is that the traditional lens was replaced by a metalens to further reduce the system size.

As mentioned above, an advantage to use a resonant device is that much less power is required to switch the device, as compared to a non-resonant device, such as an MZI. A disadvantage to resonant devices, however, is that optical power builds up in the devices making their performance sensitive to optical power. This is particularly true with silicon, as silicon rings can easily self-heat, shifting their resonance at modest optical powers. ${ }^{36}$ Another disadvantage to resonant devices is that they operate on a single wavelength of light, and the particular resonant wavelength can be very sensitive to fabrication and temperature. Each ring may require its own individual temperature sensitive setpoints for operation.

\subsubsection{MEMS waveguide switch}

In Ref. 22, a MEMS switch, rather than a ring resonator, was used to switch light from an input bus to another waveguide. This switch is described in detail in Ref. 37, and Fig. 9 illustrates how the switch functions. The switch consists of a two layer structure, with one set of waveguides suspended above the other. In the "off" or unpowered state, the suspended waveguide is far enough away from the lower waveguide that there is no optical interaction between the two layers. The light can, therefore, continue in the lower waveguide unimpeded. In the "on" state, a voltage is applied to bring the coupler section of the upper waveguide close to the lower waveguide. This causes light to transfer from the lower waveguide to the upper waveguide via an adiabatic taper in the upper waveguide. Once in the upper waveguide, the light travels through a bend, and then the light is transferred back to another waveguide in the lower layer by a similar coupler section oriented in reverse.

This switch has many favorable characteristics. It works over a wide wavelength range (demonstrated from 1460 to $1580 \mathrm{~nm})$, has low loss in the "on" position $(0.47 \mathrm{~dB})$, has very low loss in the "off" position $(0.026 \mathrm{~dB}$, primarily due to the waveguide crossings in this paper, and not inherent in the switch, itself), has fast switching times $(<1 \mu \mathrm{s})$, has simple digital operation, and consumes very little power. However, the switches required an actuation voltage of $33 \mathrm{~V}$, and this high-voltage requirement is a disadvantage of a MEMS switch.

One disadvantage of this method compared to the non-MEMS methods above is that the manufacturing of such MEMS devices is less standard than the manufacture of other silicon photonics devices. However, this does not seem to be a major limitation, and large arrays of 
(a)

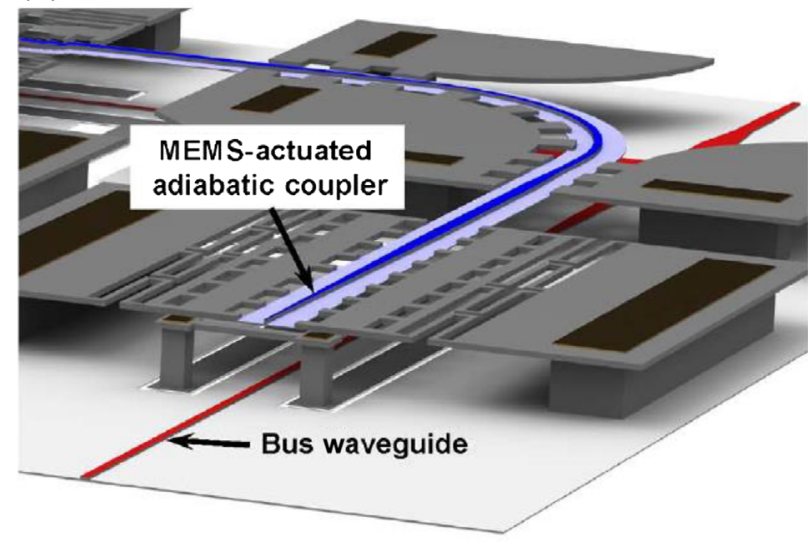

(b)

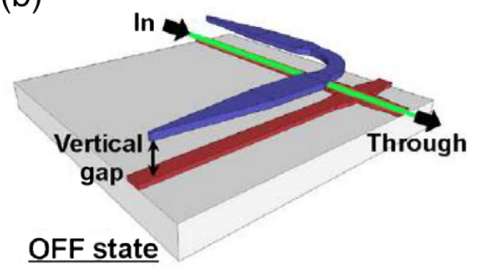

(c)

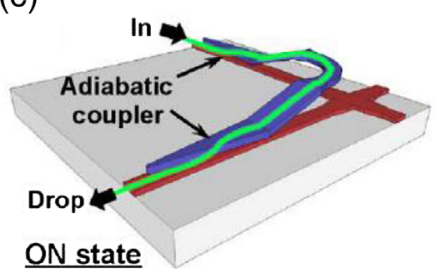

Fig. 9 MEMS waveguide switch, reproduced with permission from Ref. 37 and used in Ref. 22. (a) A diagram of switch showing the waveguides and the MEMS structure. (b) In the OFF state, the waveguides are far apart and do not interact. (c) In the ON state, adiabatic tapers are pulled toward to lower waveguide to allow light to couple.

similar switches $(240 \times 240)$ have been demonstrated, targeted for optical data-comm applications. ${ }^{37}$ Similar MEMS switches have been developed with lateral, rather than vertical couplers, and use a process more similar to a standard silicon photonics process. ${ }^{38,39}$ Another concern with MEMS devices could be long term reliability. To allay this concern, in Ref. 37, the switch was actuated for 10 billion cycles without significant degradation in optical performance.

Another limitation of this method is the size of the switch. Although more compact than an MZI-based switch, the switches are still somewhat large. In Fig. 6, the cells, each containing one switch and a grating, are on a $135-\mu \mathrm{m}$ pitch in both directions. Finally, the waveguides and switches are fabricated in silicon waveguides. The use of silicon may limit this technology to lower optical powers. However, unlike MZI and ring resonator-based switches, these switches do not rely on the phase of the light. Therefore, a non-linearity that induces only a phase shift, and not optical loss, can be tolerated. It may be possible to fabricate these devices in SiN waveguides instead of silicon, to increase the amount of optic power the waveguides can carry. However, the lower refractive index of $\mathrm{SiN}$ may require a larger bending radius, increasing the size of the device. Additionally, this would make the fabrication of the structure more complicated, as polysilicon would still be needed for other parts of the MEMS device, such as the electrodes.

\subsubsection{MEMS grating switch}

In Ref. 23, a different MEMS switch was demonstrated to enable a greater density of switches and gratings. Similar to the work in Ref. 21, this switch combines the functionality of the two components, the grating and the switch, into a single-grating switch. The switch is illustrated in Fig. 10 and works using a similar MEMS motion as the switch in Ref. 22. In the "off" position, a grating is suspended above another waveguide, so there is no interaction. In the "on" position, the grating is brought close the waveguide, and the grating functions in the usual manner to direct light out of the chip, toward the lens. In Ref. 23, a serpentine waveguide path was used to create a $10 \times 10$ array using only this one type of switch. The elements in the array were spaced by $100 \mu \mathrm{m}$ in each direction. Very little of the space between elements is taken up by the switch, itself, and the authors suggest that an array spacing of $25 \mu \mathrm{m}$ is feasible. Because of the similarities between this MEMS switch and that of waveguide MEMS switch above,$^{37}$ it is expected to have many similar properties, such as speeds, actuation voltages, and lifetimes. Although similar, the actuation voltage (at $15 \mathrm{~V}$ ) is a little lower, and the switch speed $(<4 \mu \mathrm{s})$ is a little slower than in Ref. 37. This implies that a weaker spring design was used in this grating switch compared to the design in Ref. 40. To demonstrate long-term reliability, a test grating switch was 
(a)

OFF state

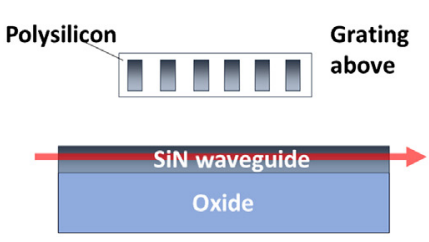

(b)

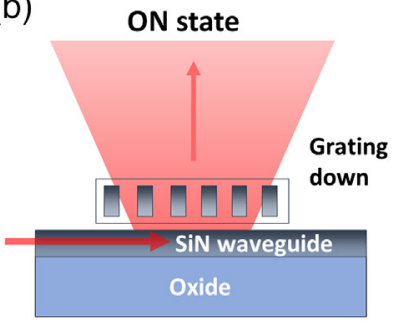

(c)

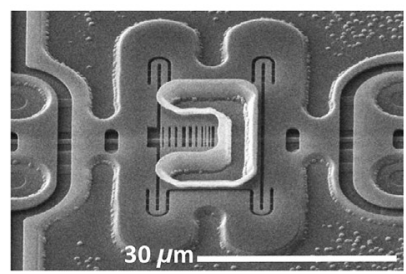

Fig. 10 MEMS grating switch, reproduced with permission from Ref. 23. (a) In the OFF state, the grating is far from the waveguide and does not interact with the waveguide. (b) The ON state is pulled toward to lower waveguide to allow light to couple, and the grating emits light from the waveguide. (c) SEM image of grating switch.

actuated for 9 billion cycles and no change in motion was observed. This was a mechanical test structure, however, and ideally the test should be repeated to verify that optical performance is also unaffected.

These devices were fabricated from $\mathrm{SiN}$ waveguides and polysilicon gratings. This material platform should enable higher optical powers and designs that operate at shorter wavelengths, even with the use of polysilicon gratings. Because the light does not need to travel though the polysilicon very far, the increased optical absorption of the polysilicon at shorter wavelengths, or higher optical powers, does not significantly impact performance. However, heating of the polysilicon grating at higher optical powers could be a concern.

The serpentine path method to creating a 2D array is not scalable to very large arrays due to the very large path length required, with realistic waveguide losses. That limitation can be overcome using a second type of switch, likely in row/column addressing format as discussed above for other switch types. This has not yet been demonstrated.

One limitation of this technique is that the grating orientation is determined by the input waveguide. This is different than in all the other examples, and in Ref. 22, the freedom to use different grating orientations was used to optimize coupling between the gratings at different locations and the lens. Even with a grating switch, different grating designs can be used at different locations, but there will be more limited design freedom.

\section{Conclusion}

LABS is a chip-based beam steering method where the light is directed by a series of switches and waveguides, from a source to an output location, most commonly a grating, on the chip. The method has a number of advantages when compared to other technologies. Like other chip-based beam-steering methods, it is expected to be amenable to low-cost manufacturing, with much more reliability and compactness than macromechanically beam-steered systems. Compared to MEMS mirrors, it enables fast random access scanning with no concerns about acoustic coupling. Compared to optical phased-arrays, control is greatly simplified.

Out of the switch types covered, here, MEMS-based switches may be the most ideal. These switches are fast, easy to control, reliable, operate over a fairly broad wavelength range and with low power. The disadvantages to the MEMS switches, however, are that they do require some non-standard processing and also require high voltages to actuate. Another switch with good properties is the ring resonator switch. Ring resonator switches are even more compact and require low power to operate. However, they can be temperature sensitive, may be limited to low optical powers, and are more difficult to control than the binary MEMS switches. The smallest switches demonstrated are the ring resonator and MEMS switches that combined the grating and switch into one compact device.

The biggest limitation of a LABS system is that the beam can only be steered to a fixed number of beam positions. It is, therefore, important that this number be as large as possible, to create a detailed LiDAR image. The MEMS grating switches and the ring resonator switches are both compact enough to produce arrays of hundreds of thousands of these devices on a single chip. 
Another method to achieve a high-resolution LiDAR image is to combine another method of steering with the switched steering technique. Typically, the other steering technique is a device (such as a grating) whose emission angle depends on the wavelength. This enables wavelength steering in one direction with switched steering in the other direction. Either the use of two steering mechanisms or a very compact switch should enable a LABS system to achieve the high number of beam positions desired in a LiDAR system.

\section{Acknowledgments}

The author would like to gratefully acknowledge the assistance of Lucas Benney, Dan Goldman, and Eugene Cook. The author would also like to thank You-Chai Chang, Josué J. López, Kan $\mathrm{Wu}$, Ming Wu, and Optica (formerly OSA) for allowing the reproduction of the prior published figures.

\section{References}

1. T. Raj et al., "A survey on LiDAR scanning mechanisms," Electronics 9(5), 741 (2020).

2. G. M. Akselrod, "Optics for automotive LiDAR: metasurface beam steering enables solidstate, high-performance LiDAR," Laser Focus World, 2019, https://www.laserfocusworld .com/optics/article/14036818/metasurface-beam-steering-enables-solidstate-highperformancelidar (accessed 11 July 2021).

3. C.-P. Hsu et al., "A review and perspective on optical phased array for automotive LiDAR," IEEE J. Sel. Top. Quantum Electron. 27(1), 1-16 (2021).

4. P. Padmanabhan, C. Zhang, and E. Charbon, "Modeling and analysis of a direct time-offlight sensor architecture for LiDAR applications," Sensors 19, 5464 (2019).

5. M. Perenzoni and D. Stoppa, "Figures of merit for indirect time-of-flight 3D cameras: definition and experimental evaluation," Remote Sens. 3, 2461-2472 (2011).

6. F. Schwarz et al., "Resonant 1D MEMS mirror with a total optical scan angle of $180^{\circ}$ for automotive LiDAR,” Proc. SPIE 11293, 1129309 (2020).

7. J. Zhou and K. Qian, "Low-voltage wide-field-of-view lidar scanning system based on a MEMS mirror," Appl. Opt. 58(5), A283-A290 (2019).

8. Y. Kim et al., "Large-area liquid crystal beam deflector with wide steering angle," Appl. Opt. 59(24), 7462-7468 (2020).

9. Y. Wang et al., "2D broadband beamsteering with large-scale MEMS optical phased array," Optica 6(5), 557-562 (2019).

10. C. V. Poulton et al., "8192-element optical phased array with $100^{\circ}$ steering range and flipchip CMOS," in Conf. Lasers and Electro-Opt., San Jose (2020).

11. J. C. Hulme et al., "Fully integrated hybrid silicon free-space beam steering source with 32-channel phased array," Proc. SPIE 8989, 898907 (2014).

12. D. Kwong et al., "On-chip silicon optical phased array for two-dimensional beam steering," Opt. Lett. 39(4), 941-944 (2014).

13. S.-H. Kim et al., "Thermo-optic control of the longitudinal radiation angle in a silicon-based optical phased array," Opt. Lett. 44, 411-414 (2019).

14. S. Saghaye-Polkoo and C. K. Renshaw, "Imaging-based beam steering for free-space optical communication," Appl. Opt. 58(13), D12-D20 (2019).

15. R. Marchetti et al., "Coupling strategies for silicon photonics integrated chips," Photonics Res. 7(2), 201-239 (2019).

16. C. Li et al., "Lens-based integrated 2D beam-steering device with defocusing approach and broadband pulse operation for Lidar application," Opt. Express 27(23), 32970-32983 (2019).

17. C. Li et al., "A cylindrical lens-based integrated 2D beam-steering device using staircase grating emitters," in Conf. Lasers and Electro-Opt., San Jose (2020).

18. H. Ito et al., "Wide beam steering by slow-light waveguide gratings and a prism lens," Optica 7(1), 47-52 (2020).

19. J. J. Lopez et al., "Planar-lens enabled beam steering for chip-scale LIDAR," in Conf. Lasers and Electro-Opt., San Jose, CA (2018). 
20. D. Inoue et al., "Demonstration of a new optical scanner using silicon photonics integrated circuit," Opt. Express 27(3), 2499-2508 (2019).

21. Y.-C. Chang et al., "Metalens-enabled low-power solid-state 2D beam steering," in Conf. Lasers and Electro-Opt., San Jose (2019).

22. X. Zhang et al., "A $20 \times 20$ focal plane switch array for optical beam steering," in Conf. Lasers and Electro-Opt., San Jose (2020).

23. E. H. Cook et al., "Polysilicon grating switches for LiDAR," J. Microelectromech. Syst. 29(5), 1008-1013 (2020).

24. H. Becker and C. J. Krückel, "Out-of-plane focusing grating couplers for silicon photonics integration with optical MRAM technology," IEEE J. Sel. Top. Quantum Electron. 26(2), $1-8$ (2020).

25. C. V. Poulton et al., "Large-scale silicon nitride nanophotonic phased arrays at infrared and visible wavelengths," Opt. Lett. 42(1), 21-24 (2017).

26. A. Michaels and E. Yablonovitch, "Inverse design of near unity efficiency perfectly vertical grating couplers," Opt. Express 26(4), 4766-4779 (2018).

27. X. Cao et al., "Lidar system based on lens assisted integrated beam steering," Opt. Lett. 45(20), 5816-5819 (2020).

28. M. W. Geis et al., "Submicrosecond submilliwatt silicon-on-insulator thermooptic switch," IEEE Photonics Technol. Lett. 16(11), 2514-2516 (2004).

29. M. R. Watts et al., "Adiabatic resonant microrings (ARMs) with directly integrated thermal microphotonics," in Conf. Lasers and Electro-Opt. and Conf. Quantum Electron. and Laser Sci. Conf., Baltimore (2009).

30. S. Saghaye-Polkoo and C. K. Renshaw, "Hybrid imaging-based beam steering system using a sparse photonic integrated circuit outcoupling array," in Conf. Lasers and Electro-Opt., San Jose (2020).

31. S. Y. Siew et al., "Review of silicon photonics technology and platform development," J. Lightwave Technol. 39(13), 4374-4389 (2021).

32. G. Takeuchi et al., "Thermally controlled Si photonic crystal slow light waveguide beam steering device," Opt. Express 26(9), 11529-11537 (2018).

33. B. E. Little et al., "Microring resonator channel dropping filters," J. Lightwave Technol. 15(6), 998-1005 (1997).

34. S. Saghaye-Polkoo and C. K. Renshaw, "Integrated photonic outcoupling array for imagingbased beam steering," in IEEE Photonics Soc. Summer Topical Meeting Ser., Ft. Lauderdale (2019).

35. G. Luo et al., "A densely integrated micro-ring optical switch network for beam steering," in Asia Commun. and Photonics Conf., Beijing (2020).

36. V. R. Almeida and M. Lipson, "Optical bistability on a silicon chip," Opt. Lett. 29(20), 2387-2389 (2004).

37. T. J. Seok et al., "Large-scale broadband digital silicon photonic switches with vertical adiabatic couplers," Optica 3(1), 64-70 (2016).

38. H. Sattari et al., "Compact broadband suspended silicon photonic directional coupler," Opt. Lett. 45(11), 2997-3000 (2020).

39. S. Han et al., " $32 \times 32$ silicon photonic MEMS switch with gap-adjustable directional couplers fabricated in commercial CMOS foundry," J. Opt. Microsyst. 1(2), 024003 (2021).

40. T. J. Seok et al., "Wafer-scale silicon photonic switches beyond die size limit," Optica 6(4), 490-492 (2019).

Steven J. Spector is a distinguished member of the technical staff and group leader at Draper, where he is leading efforts in integrated photonics, imaging, and LiDAR. He received his BSE in engineering physics from Lehigh University in 1991 and his $\mathrm{PhD}$ in physics from the Stony Brook University in 1997. Prior to joining Draper, he worked at Lincoln Laboratory from 1999 to 2015, where he led silicon photonics efforts and a number of projects in nanofabrication. 